\title{
Allopurinol Administered after Inducing Hypoxia- Ischemia Reduces Brain Injury in 7-Day-Old Rats
}

\author{
CHARLES PALMER, JAVAD TOWFIGHI, REBECCA L. ROBERTS, AND DANIEL F. HEITJAN \\ Departments of Pediatrics (C.P., R.L.R./ and Pathology (Neuropathology) /J.T./ and Center for Biostatistics and \\ Epidemiology [D.F.H.]. The Milton S. Hershey Medical Center. The Pennsylvania State University, \\ Hershey: Pennsvlvania 17033
}

\begin{abstract}
We determined that treatment of immature rats with allopurinol at $15 \mathrm{~min}$ after cerebral hypoxiaischemia reduces brain damage. Seven-d postnatal rats were subjected to right common carotid artery ligation followed by $2.25 \mathrm{~h}$ of hypoxia $\left(8 \% \mathrm{O}_{2}\right)$. At $15 \mathrm{~min}$ of recovery in room air, the rat pups received either allopurinol (135 mg/kg s.c.) or saline. Some of the rats $(n=65)$ were killed at $42 \mathrm{~h}$ of recovery for measurement of cerebral hemispheric water content. Other animals $(n=63)$ were killed at $30 \mathrm{~d}$ for morphologic assessment of the severity of damage. In separate rats, we measured the levels of allopurinol and its metabolites in serum and in the brain around the time of peak serum levels. We also determined the effect of allopurinol on rat pup body temperature. Allopurinol reduced the increase in right hemisphere water content and markedly reduced atrophy. No cavitary lesions were seen in the 31 allopurinol-treated rats, whereas 15 of 32 saline-treated rats had cavitary cerebral lesions. Histologic examination confirmed that the allopurinol-treated rats had less brain injury. Serum allopurinol and oxypurinol peaked between 0.5 and $1 \mathrm{~h}$ after allopurinol injection. Their peak serum concentrations at $0.75 \mathrm{~h}$ postinjection combined was between 360 and $510 \mu \mathrm{M}$. Allopurinol did not lower rectal temperature more than $0.04^{\circ} \mathrm{C}$. In conclusion, high-dose allopurinol administered at $15 \mathrm{~min}$ of recovery from cerebral hypoxia-ischemia markedly reduces both acute brain edema and long-term cerebral injury in immature rats. (Pediatr Res 33: 405-411, 1993)
\end{abstract}

\section{Abbreviations}

GFAP, glial fibrillary acidic protein $\mathrm{R} / \mathrm{L}$, right to left

Reperfusion of the previously ischemic brain leads to formation of cytotoxic free radicals $(1-3)$. Endothelial cells lining the cerebral blood vessels can generate superoxide via xanthine oxidase activity (4), prostaglandin metabolism (5), and damaged mitochondria (6). Allopurinol, via its active metabolite oxypurinol, inhibits xanthine oxidase (7). In animal models of cerebral ischemia, pretreatment with allopurinol or oxypurinol successfully reduces ischemic brain injury $(8-15)$. However, the ability of allopurinol pretreatment to reduce hypoxic-ischemic brain injury appears to require doses in excess of that needed to inhibit xanthine oxidase $(13,14,16)$.

In vitro studies have shown that allopurinol and oxypurinol

Received September 9. 1992: accepted December 17. 1992.

Correspondence: Dr. Charles Palmer. Department of Pediatrics. The Milton S. Hershey Medical Center. The Pennsylvania State University. P.O. Box 850. Hershey. PA 17033.

Supported by Grant NS 29704 can scavenge hydroxyl radicals and chelate transition metals in proportion to their concentration (17-19). Because free radicals may contribute to microvascular dysfunction and subsequent brain injury after cerebral ischemia $(1-3)$, we hypothesized that high-dose ailopurinoi administered after inducing cerebral hypoxia-ischemia would reduce brain injury.

We have previously reported that allopurinol administered before cerebral hypoxia-ischemia reduces brain injury in the immature rat (11). The present study, using the same model, shows that allopurinol administered $15 \mathrm{~min}$ after cerebral hypoxia-ischemia is also neuroprotective. The serum and brain levels of allopurinol and its metabolites are described.

\section{MATERIALS AND METHODS}

We induced a hypoxic-ischemic insult to the right cerebral hemisphere of 7-d postnatal rat pups. To determine early injury, we measured right hemispheric water content at $42 \mathrm{~h}$ of recovery. The extent of permanent injury was evaluated in pups that were allowed to recover to $30 \mathrm{~d}$ of age. The levels of allopurinol and its metabolites as well as temperature alterations after allopurinol injection were determined in normal 7-d-old rat pups.

Animal model. Seven-d-old Wistar (Charles River, Wilmington. MA) rat pups of either sex were taken at random from their litters and anesthetized with a mixture of halothane (4\% halothane for induction, $1-1.5 \%$ for maintenance), $30 \%$ oxygen, and the balance nitrous oxide. The right common carotid artery of each pup was permanently ligated with 4-0 surgical silk through a midline neck incision. The wound was sutured and the animals allowed to recover with their dams for $3 \mathrm{~h}$. Duration of anesthesia was less than $5 \mathrm{~min}$. After carotid ligation, the pups were numbered sequentially.

Rat pups were placed in airtight jars and exposed to a continuous flow of $8 \%$ oxygen- $-92 \%$ nitrogen gas mixture as described previously (11). The jars were partially submerged in a circulating water bath maintained at $36.8^{\circ} \mathrm{C}$ to provide a stable thermal environment. Air temperature in the middle of the jar was maintained between $33^{\circ}$ and $34^{\circ} \mathrm{C}$. After $2.25 \mathrm{~h}$ of hypoxia, the jars were opened to room air and the pups returned to their dams. At $15 \mathrm{~min}$ of recovery, pups were treated with a single s.c. injection of allopurinol $135 \mathrm{mg} / \mathrm{kg}, \mathrm{pH} 11.2$ (Zyloprim sodium, Burroughs Wellcome, Research Triangle Park, NC) or an equal volume $(0.01 \mathrm{~mL} / \mathrm{g}$ animal weight) of saline, $\mathrm{pH} 11.2$. The treatment was alternated between allopurinol and saline according to the sequential numbers. We chose the dose based on previous studies in which pretreatment with $135 \mathrm{mg} / \mathrm{kg}$ prevented brain injury from a similar insult (11).

The procedures used in this study were approved by our Committee on Animal Investigation. The investigators were blinded to treatment during all measurements of brain injury described below.

Water content. After $42 \mathrm{~h}$ of recovery with their dams, the pups $(n=65)$ were decapitated and their brains removed. A 
portion (150-200 mg) from the posterolateral section of each cerebral hemisphere was placed in a tared $5-\mathrm{mL}$ glass vial and immediately weighed on a microanalytical balance. The posterior half of the right hemisphere (ipsilateral to common carotid artery ligation) represented the area most severely injured, whereas the corresponding area in the left hemisphere served as the control. Subsequently, the specimen was desiccated at $70^{\circ} \mathrm{C}$ for $72 \mathrm{~h}$. Reweighing ascertained the dry weight of the tissue, and by subtracting dry weight from the total weight the water content of the hemisphere was obtained. Water content was determined as a percentage of the total weight (11).

Neuropathologic procedures. To examine long-term neuropathologic outcome, 64 rat pups were subjected to the combined hypoxic-ischemic insult described above. All the pups survived the 2.25-h hypoxic exposure. Pups were treated with saline or allopurinol at $15 \mathrm{~min}$ of recovery and randomized as described above. Treatment groups were identified by clipping the tip of the tail or cutting a small notch in the ear. These procedures incurred minimal bleeding.

At $30 \mathrm{~d}$ ( $23 \mathrm{~d}$ of recovery), the animals were killed with a lethal intraperitoneal dose of pentobarbital $(150 \mathrm{mg} / \mathrm{kg})$. A single saline-treated pup died during recovery. The brains were carefully removed from the skull and immersion-fixed in a mixture of formaldehyde, $1 \%$ acetic acid, and methanol, (1:1:8 vol/vol).

Gross neuropathologic grading. A pair of examiners together allocated the whole brains into normal brains and brains with mild, moderate, or severe categories of damage. The choice of category was based on inspection of the right cerebral hemisphere size (ipsilateral to ligation) compared with the left (contralateral to ligation). Normal referred to no difference in size; mild referred to some discrepancy in size, the right being smaller than the left, without any visible cavitary lesion; moderate indicated discrepancy in size due to a cavitary lesion in the right hemisphere; and severe related to extensive cystic infarction of the right hemisphere with almost total destruction of its posterolateral aspect. These categories of damage have been previously illustrated (11).

Brain morphometry (interhemispheric diameter ratio determination). To assess the degree of right hemisphere atrophy, we used the interhemispheric diameter ratio. Accordingly, a $2-\mathrm{mm}$ coronal slice was cut at the mid-mammillary body level and the diameter of each cerebral hemisphere was measured from the midpoint of a straight line connecting the interhemispheric fissure to the midmammillary region under a dissecting microscope. This coronal level was chosen because it approximated the region of maximal injury. Then the ratio of the $\mathrm{R} / \mathrm{L}$ hemisphere diameter was calculated and expressed as a percentage. We also determined the $\mathrm{R} / \mathrm{L}$ hemisphere diameter ratio on eight normal 30-d-old rats that had not been exposed to hypoxia or carotid artery ligation to determine the range of normal interhemisphere assymetry.

Histopathologic scoring of damage. To accurately differentiate between normal (undamaged) brains and mildly damaged brains, we developed a method for histologic scoring of damage. For this study, brains obviously damaged with cavitary lesions on gross inspection were excluded from histologic scoring. This left all 31 allopurinol-treated and 17 saline-treated brains for histologic examination. Accordingly, the coronal slices used for determination of the interhemisphere diameter ratio were processed for paraffin embedding, and $6-\mu \mathrm{m}$ sections at the level of the infundibulum were obtained. The slides were stained with hematoxylin and eosin, and for GFAP they were stained using an immunoperoxidase technique and counterstained with hematoxylin. The polyclonal GFAP antibody was produced in rabbits against cow spinal cord (Dako Corp., Carpenteria, CA). Reactive astrocytosis is a sensitive indicator of long-standing brain damage and is readily identified with this technique (20).

To determine the normal appearance of astrocyte staining, we examined sections from eight normal (nonligated and nonhypoxic) rats. With this technique, fixed normal brains showed only weak astrocyte staining predominantly in the corpus cal- losum and internal capsule. Strong staining was observed only in reactive astrocytes within damaged areas. Reactive gliosis was defined as a focal aggregation of cells that stained strongly for GFAP. In contrast to the normal brains, astrocytes in regions of reactive gliosis were increased in number and staining intensity.

Each hemisphere was divided into 11 anatomical regions (Fig. 1) that were individually examined and a point was assigned for the presence of reactive gliosis. The histopathologic score ranged from 0 to 11 and represented the sum of the damaged regions per hemisphere. Normal brains and hemispheres always scored 0 because a point was only allotted for a region that had a focus of reactive gliosis.

Measurement of allopurinol and metabolite levels. Seven-d-old rat pups were injected s.c. with $135 \mathrm{mg} / \mathrm{kg}$ of allopurinol and decapitated at intervals ranging from 15 min to $6 \mathrm{~h}$. Blood was collected from the severed neck vessels and the serum deproteinated with $30 \%$ perchloric acid in a $10 \% \mathrm{vol} / \mathrm{vol}$ ratio. The head was immediately frozen in liquid nitrogen and stored at $-70^{\circ} \mathrm{C}$. The brain was dissected from the skull in a cold box at $-20^{\circ} \mathrm{C}$. A sample of tissue from the cerebral hemisphere $( \pm 100 \mathrm{mg})$ was powdered and weighed in the cold box, then extracted into perchloric acid as previously described (21). The brain extracts were analyzed using HPLC as modified from Wung et al. (22). We used a Waters-Bondapak C-18 reverse phase column (Waters Associates, Milford, MA) fitted with a C-18 precolumn filter module. The mobile phase consisted of $50 \mathrm{mM}$ potassium phosphate, $\mathrm{pH} 6.0$, with $1 \%$ methanol vol/vol, at a flow rate of 1.4 $\mathrm{mL} / \mathrm{min}$. Peaks were detected at $254 \mathrm{~nm}$ and their identity verified by retention time, coelution with known standards, and by matching the UV absorbance spectra of each peak with authentic standards for allopurinol, oxypurinol, and allopurinol

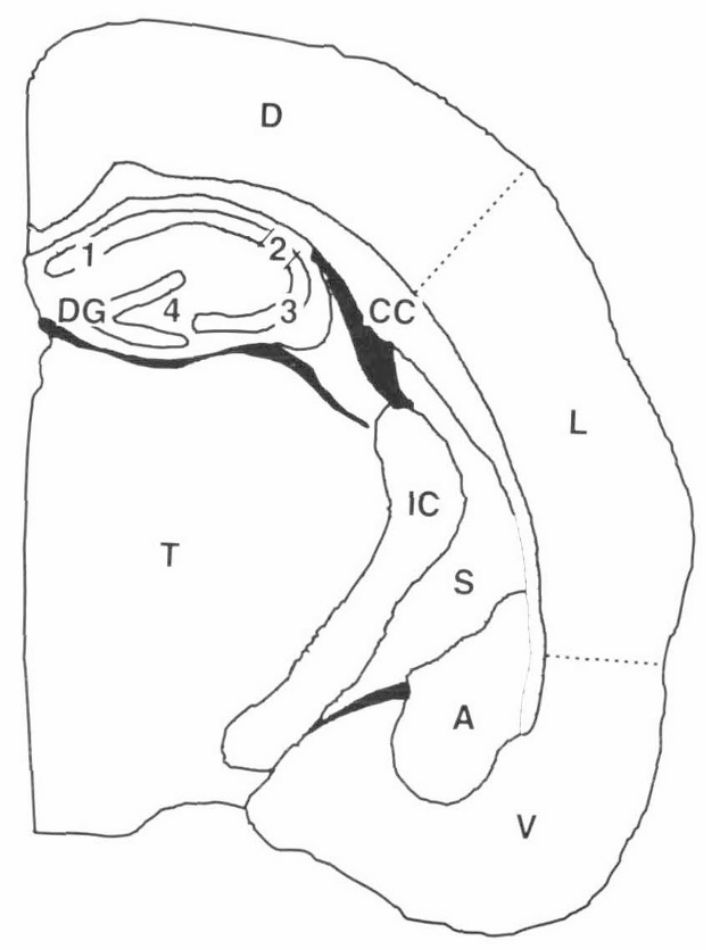

Fig. 1. Coronal section of right hemisphere. The drawing of the right hemisphere from a representative coronal brain section used to determine the histopathology score is subdivided into the following regions: $D$, dorsal cortex; $L$, lateral cortex; $V$, ventral cortex; $l, 2,3$, and 4 , CA1, $\mathrm{CA} 2, \mathrm{CA} 3$, and $\mathrm{CA} 4$ regions of the hippocampus, respectively; $D G$, dentate gyrus; $T$, thalamus; $S$, striatum; $A$, amygdaloid nucleus; $C C$, corpus callosum; and $I C$, internal capsule. The CC and IC were not part of the 11 regions composing the score. The ventral cortex was the cortex below the rhinal fissure; the remaining cortex above the rhinal fissure was divided equally into lateral and dorsal portions. 
riboside using a Hewlett-Packard 1090 photodiode-array detector (Hewlett-Packard Co., Palo Alto, CA).

Effect of allopurinol on rat pup body temperature. Rat pups (nonligated and nonhypoxic) that were feeding from their dams were removed from the nest and fitted with a rectal temperature probe (511, Yellow Springs Instrument Co., Yellow Springs, $\mathrm{OH})$. They were positioned into plastic syringe barrels housed within a neonatal incubator set at $33.5^{\circ} \mathrm{C}$. This temperature was chosen because it resembled the average nesting temperature. Ninety min were required for the rectal temperature to stop fluctuating by more than $0.2^{\circ} \mathrm{C}$ in $5 \mathrm{~min}$. Then, three temperature recordings for each pup were obtained over $30 \mathrm{~min}$ and the average of the three temperatures represented the "predrug" temperature. The pups were then injected with the experimental dose of saline or allopurinol and given another 90-min equilibration period. Three temperature measurements were obtained over the next $30 \mathrm{~min}$ and averaged to form the "postdrug" temperature. The effect of the drug was determined by the difference between postdrug and predrug temperatures.

Statistical methods. We used the two-tailed $t$ test, Cochran trend test, and Mann-Whitney $U$ test where appropriate; $p<$ 0.05 was considered significant.

\section{RESULTS}

Water content. Two rat pups received allopurinol $(n=41)$ for every one that was treated with saline $(n=24)$. No pups died during the 42-h recovery period of either treatment group. The left hemisphere (contralateral to ligation) water content for the saline-treated pups was $87.92 \pm 0.22 \%$, and for the allopurinoltreated pups it was $87.80 \pm 0.30 \%$ (mean $\pm \mathrm{SD}$ ). These results are not statistically different and fall within the normal range as previously determined in 12 untreated, normal, 7-d-old rat pups (11). The left hemisphere water contents were combined to form a normal (mean $\pm 2 \mathrm{SD}$ ) reference range against which the distribution of water content in the edematous right hemisphere is illustrated (Fig. 2). Twenty-six of 41 allopurinol-treated brains had a normal right hemisphere water content. The distribution of the elevated water contents in the remaining 15 can be seen in Figure 2. In contrast, the right hemisphere water content of the 24 saline-treated pups was $91.70 \pm 0.95 \%$ (mean \pm SD); only one of the 24 saline-treated pups had a right hemisphere water content within the normal range. The right hemisphere water content of the allopurinol-treated rat pups was significantly less than that of the saline-treated pups $[p<0.0001$ (Mann-Whitney $U$ test)].

Gross neuropathology: In those pups allowed to recover until 30 postnatal $\mathrm{d}$, brain abnormalities ranged from atrophy of the right cerebral hemisphere to cystic destruction (cavitary lesions) of that hemisphere. The results are illustrated in Table 1.

Significantly less damage was seen in the allopurinol-treated pups. Twenty-four of 32 saline-treated rats developed gross brain injury. Nine were considered mild, eight moderate, and seven severe. In contrast, only 15 of the 31 allopurinol-treated appeared damaged, all of which were graded as mild. None had cavitary lesions. Statistical analysis showed that allopurinol significantly reduced the amount of atrophy and prevented cystic infarction $[p<0.001$ (Cochran trend test) $]$

Brain morphometry (interhemisphere diameter ratio). The ratio of the $\mathrm{R} / \mathrm{L}$ hemisphere diameter for eight normal 30-d-old rat pups (not subjected to carotid ligation or hypoxia) was $97 \pm$ $2.6 \%$ (mean $\pm \mathrm{SD}$ ). Therefore, the normal range for hemisphere asymmetry that would include roughly $95 \%$ of normal brains is 91.8 to $102.2 \%$ (mean \pm 2 SD). Accordingly, a ratio less than $91.8 \%$ can be regarded as abnormal and indicative of atrophy or infarction. The normal range (mean $\pm 2 \mathrm{SD}$ ) is illustrated by the striped area in Figure 3, which shows the distribution of atrophy for the experimental animals.

Damage in the 32 saline-treated brains was biphasic. Seventeen brains had an $\mathrm{R} / \mathrm{L}$ hemispheric diameter ratio greater than $70 \%$.
These brains did not have cavitary lesions. Of the remaining 15 brains, all had cavitary lesions and the diameter of the right hemisphere was less than $65 \%$ of the left $(\mathrm{R} / \mathrm{L}<65 \%)$. In contrast, all 31 allopurinol-treated brains had an $\mathrm{R} / \mathrm{L}$ hemisphere ratio greater than $70 \%$ and none had cavitary lesions. Atrophy was markedly less in the allopurinol-treated rats $[p<0.001$ (Mann-Whitney $U$ test)].

Histopathology: All the brains without cavitary lesions were examined histologically. No abnormalities were seen in the left cerebral hemispheres. Because none of the allopurinol-treated brains had cavitary lesions, all 3 I were examined histopathologically for the presence of reactive gliosis and histologic scoring of damage. One allopurinol-treated brain section, with an $\mathrm{R} / \mathrm{L}$ hemisphere diameter ratio of $99.32 \%$, was excluded from examination due to the suboptimal quality of the histologic sections. The brains of $19(63 \%)$ of the 31 allopurinol-treated rats were undamaged. In contrast, only eight of $32(25 \%)$ salinetreated brains were undamaged, because 15 had cavitary lesions and nine had reactive gliosis. Thus, gross and microscopic neuropathologic evaluation confirmed that damage was less in the allopurinol-treated brains $[p<0.001$ (Cochran trend test)] (Table 2).

I wenty brains ( 1 i ailopurinoi-treated and nine saline-treated) had between one and 11 regions of reactive gliosis. Allopurinol did not alter the regional susceptibility to injury from that seen in the saline-treated pups. The lateral CA 3 zone of the hippocampus was the most consistently involved region (20 of 20 ) in both treatment groups and in two animals was the only region damaged. The CA2 zone was damaged in 18 of 20 , whereas the CA1 region was damaged in only 14 of 20 animals. The lateral cortex and thalamus was damaged in 15 of 20 animals. The white matter, including the corpus callosum and internal capsule, was consistently spared (in brains without cavitary lesions).

There was a very good correlation $\left(\mathrm{R}^{2}=0.88\right)$ between the $\mathrm{R} /$ $\mathrm{L}$ hemisphere diameter ratio and histopathologic score. Brain injury (reactive gliosis) was seen only in those brains with an R/ $\mathrm{L}$ diameter ratio $<95 \%$. This included three brains considered normal morphometrically.

Allopurinol and metabolite levels. Allopurinol administered at $135 \mathrm{mg} / \mathrm{kg}$ s.c. peaked in the serum at $340-480 \mu \mathrm{M}(46.3-64.5$ $\mu \mathrm{g} / \mathrm{mL})$ at $0.5-0.75 \mathrm{~h}$ postinjection. Thereafter, the levels declined, with a serum half-life of $52 \mathrm{~min}$. Brain levels of allopurinol were approximately one fourth of the peak serum levels. A level of $77 \mu \mathrm{M}(10.5 \mu \mathrm{g} / \mathrm{mL})$ was attained between 0.5 and $0.75 \mathrm{~h}$ postinjection (see Fig. 4).

The serum levels of oxypurinol, the active metabolite of allopurinol, peaked $0.75-3 \mathrm{~h}$ after allopurinol injection at $21-27 \mu \mathrm{M}$ $(3.2-4.1 \mu \mathrm{g} / \mathrm{mL})$ and then declined, with a half-life of $170 \mathrm{~min}$. Brain oxypurinol levels, $15 \mu \mathrm{M}(2.2 \mu \mathrm{g} / \mathrm{mL})$, were similar to the serum levels at $0.5 \mathrm{~h}$ after allopurinol injection. The metabolite of allopurinol present in highest concentration was allopurinol riboside, which reached a maximum serum concentration of 143-175 $\mu \mathrm{M}(38.4-47.11 \mu \mathrm{g} / \mathrm{mL})$ at $1-2 \mathrm{~h}$ postinjection.

Temperature effects. To ascertain if allopurinol induced hypothermia. the postdrug temperature measurement was subtracted from the predrug measurement in four allopurinoltreated and four saline-treated pups. In the allopurinol-treated pups, the temperature difference was $0.09 \pm 0.32^{\circ} \mathrm{C}$, whereas in the saline-treated pups the temperature difference was $0.16 \pm$ $0.18^{\circ} \mathrm{C}$ (mean $\left.\pm \mathrm{SD}\right)$. Thus, only minor temperature fluctuations of a similar degree in response to either treatment were observed.

\section{DISCUSSION}

This study shows that allopurinol protects 7 -d-old rat pups from hypoxic-ischemic injury even when the drug is administered $15 \mathrm{~min}$ after the insult. The effectiveness of treatment during recovery ("rescue therapy") supports the concept that brain damage evolves during resuscitation ("secondary damage"). 

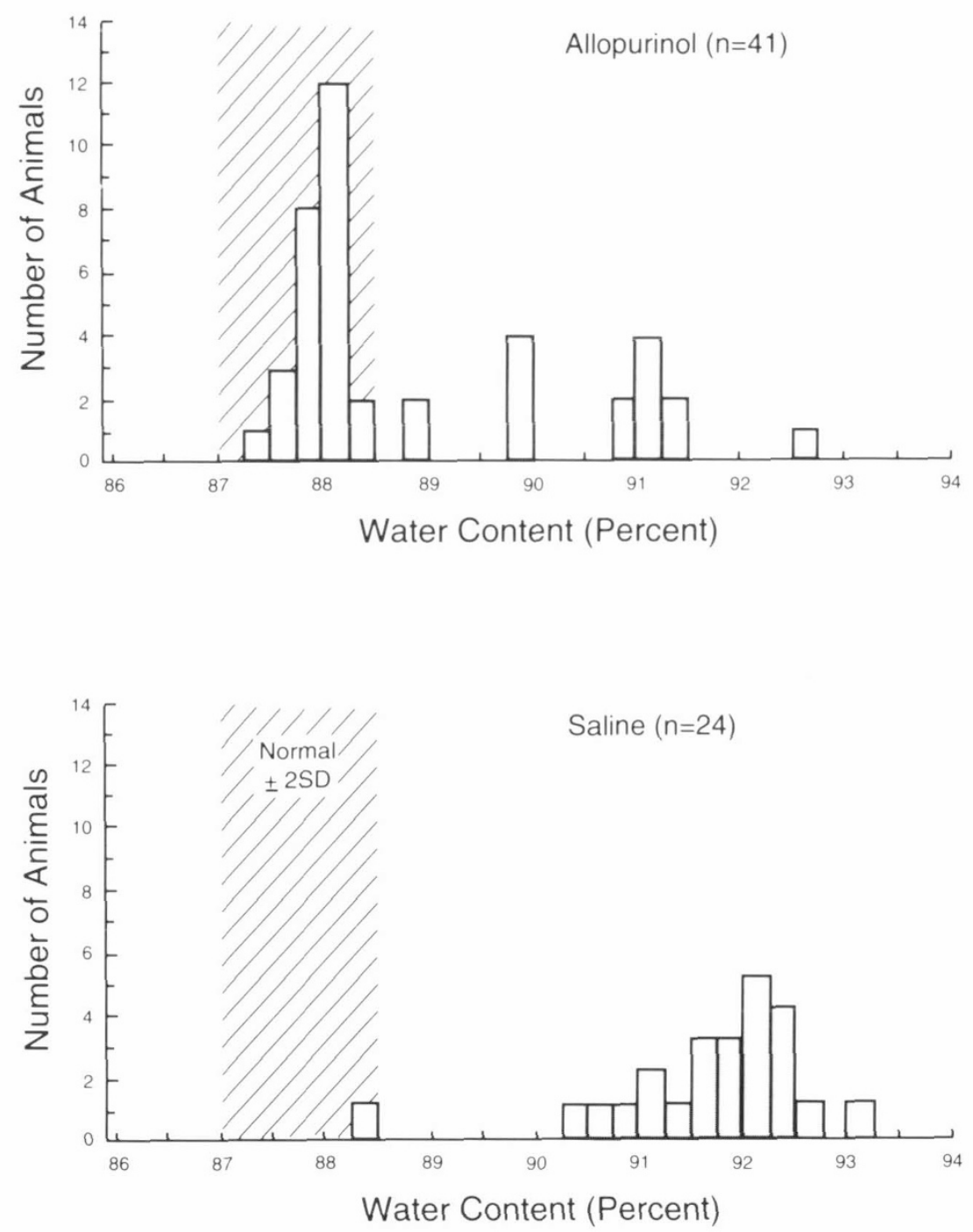

Fig. 2. Right hemisphere water content at $42 \mathrm{~h}$ of recovery after hypoxia-ischemia. The normal range (mean $\pm 2 \mathrm{SD}$ ) for cerebral water content is illustrated. Pups were treated at $15 \mathrm{~min}$ after hypoxic-ischemic insult with allopurinol $135 \mathrm{mg} / \mathrm{kg}$ s.c. or saline. The allopurinol-treated pups had lower right hemisphere water contents than the saline-treated pups. $p<0.0001$ (Mann Whitney $U$ ).

Table 1. Gross neuropathologic grading*

Treatment group (no. of animals)

\begin{tabular}{ccc}
$\begin{array}{c}\text { Gross pathologic } \\
\text { category }\end{array}$ & $\begin{array}{c}\text { Allopurinol } \\
(n=31)\end{array}$ & $\begin{array}{c}\text { Saline } \\
(n=32)\end{array}$ \\
\hline Normal & 16 & 8 \\
Mild & 15 & 9 \\
Moderate & 0 & 8 \\
Severe & 0 & 7 \\
\hline
\end{tabular}

${ }^{*} p<0.0001$ (Cochran trend test). Neuropathologic grading by a team of two examiners.

Because this is the first study to show that allopurinol administered during recovery reduces hypoxic-ischemic brain damage in any age animal, it could be possible that this neuroprotective effect is unique to the immature brain. However, studies in adult gerbils showed that oxypurinol ( $40 \mathrm{mg} / \mathrm{kg}$ intraperitoneally) administered $30 \mathrm{~min}$ after transient cerebral ischemia reduced the increased locomotor activity that is associated with hippocampal injury (23). Although our study confirms that high-dose allopurinol rescue therapy is neuroprotective, it does not address mechanisms directly apart from suggesting that this beneficial effect is not mediated through hypothermia. We monitored the effect of allopurinol on core body temperature because core body temperature closely reflects brain temperature in this model (24) and because mild hypothermia $\left(3-6^{\circ} \mathrm{C}\right)$, even during recovery from cerebral hypoxia-ischemia, can be protective (25-27).

Allopurinol's neuroprotective mechanism has usually been attributed to its ability to inhibit xanthine oxidase. In the brain. xanthine oxidase is precariously concentrated within endothelial cells $(28,29)$. This may subject the blood-brain barrier to free radical attack. Patt et al. (30) showed that inhibition of xanthine oxidase activity with a tungsten-enriched diet reduced edema and hydrogen peroxide production in the postischemic gerbil brain. They also showed that brain edema and hydrogen peroxide production correlated with xanthine oxidase activity. Liu et al. (31) showed that infarct volume in adult rats undergoing bilateral carotid occlusion was reduced by pretreatment with superoxide dismutase and catalase conjugated to polyethelylene glycol. Because these substrate-specific conjugated enzymes do not cross the blood-brain barrier (32), they are thought to scavenge superoxide and hydrogen peroxide from within cerebral blood vessels. This implicates free radical-induced microvascular injury in the pathogenesis of cerebral infarction.

Recently, the role of xanthine oxidase as the major source of endothelial cell-derived superoxide during cerebral reperfusion has been questioned. Terada et al. (28) showed that cultured endothelial cells from bovine cerebral microvessels sponta- 

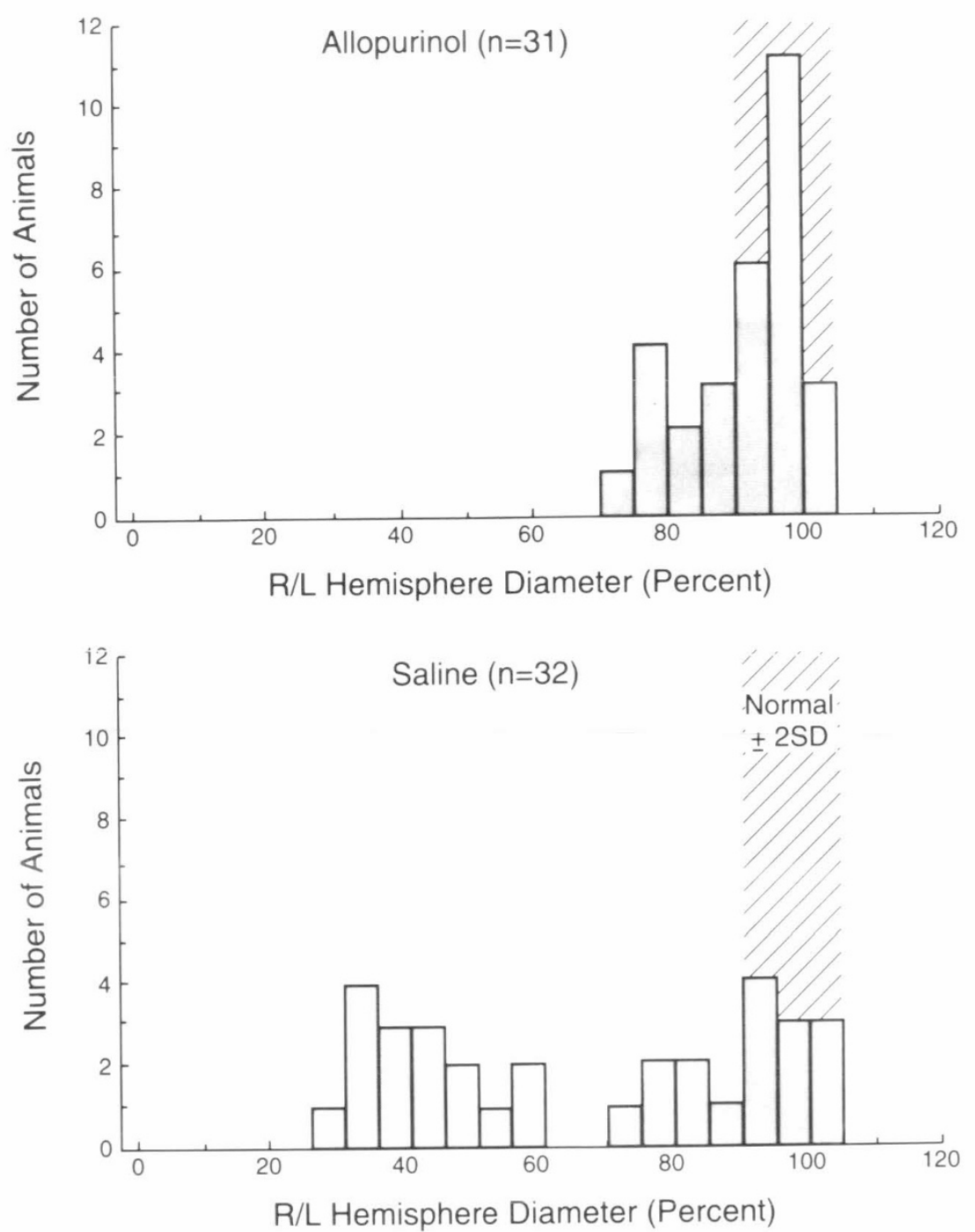

Fig. 3. Right hemisphere atrophy determined morphometrically by the R/L hemisphere diameter ratio. The normal range (mean \pm 2 SD) of hemisphere asymmetry is illustrated. Pups were treated at $15 \mathrm{~min}$ after hypoxia-ischemia with allopurinol $135 \mathrm{mg} / \mathrm{kg}$ s.c. or saline. A ratio less than $65 \%$ was associated with cavitary lesions. The allopurinol-treated pups were markedly less damaged than the saline-treated pups and had no cavitary lesions. $p<0.001$ (Mann Whitney $U$ ).

Table 2. Combined gross and microscopic assessment of established brain damage*

\begin{tabular}{lcc}
\hline \multirow{2}{*}{ Assessment } & \multicolumn{2}{c}{$\begin{array}{c}\text { Treatment group (no. } \\
\text { of animals) }\end{array}$} \\
\cline { 2 - 3 } & $\begin{array}{c}\text { Allopurinol } \\
(n=31)\end{array}$ & $\begin{array}{c}\text { Saline } \\
(n=32)\end{array}$ \\
\hline Gross damage (cavitary lesions) & 0 & 15 \\
Microscopic damage (reactive gliosis) & 11 & 9 \\
Undamaged & 20 & 8 \\
\hline
\end{tabular}

${ }^{*} p<0.0001$ by the Cochran trend test, suggesting that the distribution of damage is shifted toward more severe damage in the saline group relative to the allopurinol group.

neously secrete superoxide. However, superoxide secretion was only partially repressed when xanthine oxidase activity was inhibited. Lindsay et al. (13) reduced infarct volume in a rat model of permanent middle cerebral artery occlusion with allopurinol pretreatment but needed doses in excess of that required to inhibit xanthine oxidase. Similar findings were obtained by Betz et al. (14). These studies suggest that xanthine oxidase inhibition does not fully explain the neuroprotective mechanism of highdose allopurinol pretreatment.
We showed recently in the same neonatal rat pup model of cerebral hypoxia-ischemia that brain energy metabolism during the insult is preserved by high-dose allopurinol pretreatment (33). Therefore, pretreatment with high-dose allopurinol may reduce the severity of the hypoxic-ischemic insult. In a preliminary report, Chemtob et al. (34) pretreated newborn pigs with $140 \mathrm{mg} / \mathrm{kg}$ allopurinol and showed that allopurinol reduced postasphyxial cerebral hypoperfusion. Previous studies have suggested that allopurinol has a vasodilatory action $(35,36)$. Clearly, the effectiveness of allopurinol in this study cannot be attributed to any attenuation of the primary hypoxic-ischemic insult, inasmuch as it was given 15 min into recovery.

The near 4-fold concentration of allopurinol in the serum compared with brain homogenate suggests that allopurinol exerts dose-dependent effects from within blood vessels. Because the regional pattern of blood flow changes after hypoxia-ischemia has not been completely characterized in this model, it is still possible that allopurinol given at $15 \mathrm{~min}$ of recovery in some way prevented microvascular dysfunction and secondary ischemia. Prevention of microvascular injury and secondary infarction could explain why cavitary lesions did not occur in the brains of the allopurinol-treated pups and why, in contrast, 15 of 32 saline-treated animals had cavitary lesions.

It is possible that posttreatment (rescue therapy) with allopu- 

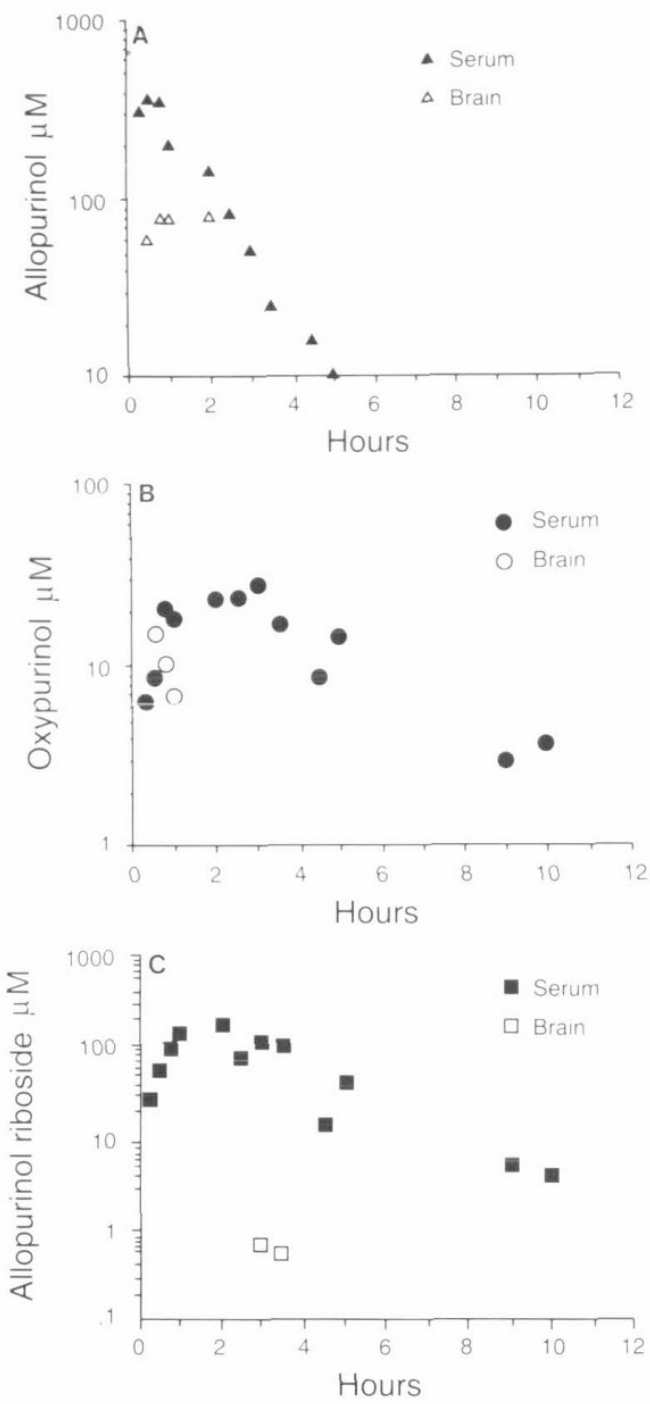

Fig. 4. Drug concentrations. Pups were injected with allopurinol 135 $\mathrm{mg} / \mathrm{kg}$ s.c. at time 0 . Serum (filled symbols) and brain (open symbols) concentrations of allopurinol $(A)$ and its metabolites oxypurinol $(B)$ and allopurinol riboside $(C)$ were later measured at intervals. The points represent the average of two to six animals.

rinol may have a different mechanism of action compared with pretreatment. Allopurinol and oxypurinol both scavenge hydroxyl radicals and chelate transition metals in a dose-dependent manner. However, concentrations in the 0.5 to $1-\mathrm{mM}$ range are required (17-19). Serum levels of allopurinol and its active metabolite oxypurinol peaked between $30 \mathrm{~min}$ and $1 \mathrm{~h}$ after injection. The serum allopurinol levels were nearly four times higher than brain levels. By combining the peak serum levels of allopurinol and oxypurinol, a concentration of approximately $0.5 \mathrm{mM}$ was achieved in the first hour after injection. This level may be sufficient to exert a direct antioxidant effect, although the ability of allopurinol to increase plasma antioxidant capacity in vivo still needs to be demonstrated. At the serum levels attained in this study, allopurinol can facilitate mitochondrial electron transport in the respiratory chain (37). In addition, allopurinol can inhibit neutrophil lysosomal enzyme release at concentrations from 0.01 to $1 \mathrm{mM}$ (38). The high serum levels of allopurinol and oxypurinol suggest that their site of action is within blood vessels. They would also have acted in the parenchyma, if a damaged blood-brain barrier allowed extravasation of intravascular contents.

In our study, more allopurinol was converted to allopurinol riboside than to oxypurinol. This is consistent with a report by
Nelson and Elion (39), who showed that animals given large doses of allopurinol metabolize relatively little to oxypurinol in comparison to allopurinol riboside. We have not found that allopurinol riboside shares allopurinol's neuroprotective effect (unpublished data). Oxypurinol, however, administered at the same time postinsult and at the same dose as allopurinol was administered in this study, also prevents brain injury (40).

Oxypurinol may have advantages over allopurinol as a rescue therapy. Oxypurinol is a better free radical scavenger than allopurinol (41); it scavenges hypochlorous acid, whereas allopurinol does not (42). It has a longer half-life and it noncompetitively inhibits xanthine oxidase. Its activity is not inhibited by the high levels of hypoxanthine and xanthine that accumulate with ischemia (41). In addition, oxypurinol does not release a superoxide radical during its metabolism as allopurinol does $(41,43)$.

In conclusion, this study shows that allopurinol rescue therapy administered $15 \mathrm{~min}$ after cerebral hypoxia-ischemia in neonatal rats reduces brain edema, selective neuronal necrosis, and cystic infarction. This study has important clinical implications for the management of hypoxic-ischemic (asphyxiated) newborn infants. Additional studies are needed to define the neuroprotective mechanisms and therapeutic time-dose window of allopurinol rescue therapy.

Acknowledgments. The authors thank Cathy Housman for preparing the histologic sections and Tina Eberly for typing the manuscript.

\section{REFERENCES}

1. Traystman RJ, Kirsch JR. Koehler RC 1991 Oxygen radical mechanisms of brain injury following ischemia a 1195

2. Kirsch JR. Helfaer MA. Lange DG. Traystman RJ 1992 Evidence for free radical mechanisms of brain injury resulting from ischemia/reperfusioninduced events. J Neurotrauma 9:S157-S163

3. Siesjo BK, Agardh C-D, Bengtsson F 1989 Free radicals and brain damage Cerebrovasc Brain Metab Rev 1:165-211

4. McCord JM 1985 Oxygen-derived free radicals in postischemic tissue injury. N Engl J Med 312:159-163

5. Kukreja RC. Kontos HA, Hess ML. Ellis EF 1986 PGH synthase and lipoxygenase generate superoxide in the presence of NADH or NADPH. Circ Res 59:612-619

6. Boveris A, Chance B 1973 The mitochondrial generation of hydrogen peroxide. Biochem J 134:707-716

7. Kellogg EW, Fridovich I 1975 Superoxide, hydrogen peroxide and singlet oxygen in lipid peroxidation by a xanthine oxidase system. J Biol Chem 250:8812-8817

8. Itoh T, Kawakami M. Yamauchi Y, Shimizu S, Nakamura M 1986 Effect of allopurinol on ischemia and reperfusion-induced cerebral injury in spontaneously hypertensive rats. Stroke 17:1284-1287

9. Martz D, Rayos G, Schielke GP. Betz AL 1989 Allopurinol and dimethylthiourea reduce brain infarction following middle cerebral artery occlusion in rats. Stroke 20:488-494

10. Pigott JP, Donovan DL. Fink JA, Sharp WV 1988 Experimental pharmacologic cerebroprotection. J Vasc Surg 7:625-630

11. Palmer C. Vannucci RC. Towfighi J 1990 Reduction of perinatal hypoxicischemic brain damage with allopurinol. Pediatr Res 27:332-336

12. Lin Y. Phillis JW 1991 Oxypurinol reduces focal ischemic brain injury in the rat. Neurosci Lett 126:187-190

13. Lindsay S, Liu T-H, Xu J, Marshall PA. Thompson JK, Parks DA, Freeman BA. Hsu CY, Beckman JS 1991 Role of xanthine dehydrogenase and oxidase in focal cerebral ischemic injury to rat. Am J Physiol 261:H2051-H2057

14. Betz AL, Randall J, Martz D 1991 Xanthine oxidase is not a major source of free radicals in focal cerebral ischemia. Am J Physiol 260:H563-H568

15. Mink RB, Dutka AJ, Hallenbeck JM 1991 Allopurinol pretreatment improves evoked response recovery following global cerebral ischemia in dogs. Stroke 22:660-665

16. Palmer C. Smith MB, Williams GD, Roberts RL. Heitjan DF 1991 Allopurinol preserves cerebral energy metabolism during perinatal hypoxic-ischemic injury and reduces brain damage in a dose dependent manner. J Cereb Blood Flow Metab 11:S144(abstr)

17. Ko KM. Godin DV 1990 Inhibition of transition metal ion-catalyzed ascorbate oxidation and lipid peroxidation by allopurinol and oxypurinol. Biochem Pharmacol 40:803-809

18. Das DK. Engelman RM. Clement R. Otani H. Prasad MR. Rao PS 1987 Role of xanthine oxidase inhibition as free radical scavenger: a novel mechanism of action of allopurinol and oxypurinol in myocardial salvage. Biochem Biophys Reś Commun 148:314-319

19. Moorhouse PC, Grootveld M. Halliwell B, Quinlan JG. Gutteridge JMC 1987 
Allopurinol and oxypurinol are hydroxyl radical scavengers. FEBS Lett 213:23-28

20. Towfighi J. Yager JY. Housman C. Vannucci RC 1991 Veuropathy of remote hypoxic-ischemic damage in the immature rat. Acta Neuropathol (Berl) 81:578-587

21. Palmer C. Brucklacher RM. Christensen MA. Vannucci RC 1990 Carbohydrate and energy metabolism during the evolution of hvpoxic-ischemic brain damage in the immature rat. J Cereb Blood Flow Metab 10:227-235

22. Wung WE. Howell SB 1980 Simultaneous liquid chromatography of 5-fluorouracil. uridine. hypoxanthine, xanthine. uric acid. allopurinol, and oxypurinol in plasma. Clin Chem 26:1704-1708

23. Helfman C. Phillis JW 1989 Oxypurinol administered post-ischemia prevents brain injury in the gerbil. Med Sci Res 17:969-970

24. Yager JY Vannucci RC 1991 Protective effects of mild hypothermia on hypoxic-ischemic brain damage in the immmature rat. Pediatr Res 29:366 A(abstr)

25. Young RSK. Olenginski TP. Yagel SK. Towfighi J 1983 The effect of graded hypothermia on hypoxic-ischemic brain damage: a neuropathologic study in the neonatal rat. Stroke 14:929-933

26. Chopp M. Chen H. Dereski MO. Garcia JH 1991 Mild hypothermic intervention after graded ischemic stress in rats. Stroke 22:37-43

27. Busto R. Dietrich WD, Globus MY-T, Valdes I, Scheinberg P. Ginsberg MD 1987 Small differences in intraischemic brain temperature critically determine the extent of ischemic neuronal injury. J Cereb Blood Flow Metab 7:729-738

28. Terada LS, Willingham IR. Rosendich ME. Leff JA, Kindt GW. Repine JE 1991 Generation of superoxide anion by brain endothelial cell xanthine uxidase. J Cell Physiò 148:191-196

29. Betz AL 1985 Identification of hypoxanthine transport and xanthine oxidase activity in brain capillaries. J Neurochem 44:574-579

30. Patt A. Harken AH. Burton LK. Rodell TC. Piermattei D. Schorr WJ, Parker NB. Berger EM. Horesh IR. Terada LS. Linas SL. Cheronis JC. Repine JE 1988 Xanthine oxidase-derived hydrogen peroxide contributes to ischemia reperfusion-induced edema in gerbil brains. J Clin Invest 81:1556-1562
31. Liu TH, Beckman JS. Freeman BA, Hogan EL. Hsu CY 1989 Polyethylene glycol-conjugated superoxide dismutase and catalase reduce ischemic brain injury. Am J Physiol 256:H589-H593

32. Haun SE. Kirsch JR. Helfaer MA. Kubos KL. Traystman RJ 1991 Polvethylene glycol-conjugated superoxide dismutase fails to augment brain superoxide dismutase activity in piglets. Stroke 22:655-659

33. Williams GD. Palmer C. Heitjan DF. Smith MB 1992 Allopurinol preserves cerebral energy metabolism during perinatal hypoxia-ischemia: a ${ }^{3 i} \mathrm{P}$ NMR study in unanesthetized immature rats. Neurosci Lett 144:103-106

34. Chemtob S. Parys R. Bordewick D. Fernandez H. Barna T. Varma DR 1992 Lazaroids and allopurinol prevent post-asphyxial decrease in cerebral blood flow and hasten recovery of brain electrophysiologic function in the newborn pig. Pediatr Res 31:28A(abstr)

35. Ellis CH. Touw KB. Dickerson SW 1973 Some acute hemodynamic effects of large doses of sodium allopurinol in open-chested dogs. Arch Int Pharmacodyn 205:355-367

36. Arnold WL. DeWall RA. Kezdi P. Zwart HHJ The effect of allopurinol on the degree of early myocardial ischemia. Am Heart J 99:614-624

37. Peterson DA. Kelly B. Gerrard JM 1986 Allopurinol can act as an electron transfer agent. Is this relevant during reperfusion injury? Biochem Biophys Res Commun 137:77-79

38. Mikulikova D. Bosmansky K. Bosak V. Ondrasik M 1989 The effect of allopurinol on lysosomal enzyme release. Z Rheumatol 48:26-29

39. Nelson DJ. Elion GB 1984 Metabolic studies of high doses of allopurinol in humans. Adv Exp Med Biol 165:167-170

40. Palmer C. Roberts RL 1991 Reduction of perinatal brain damage with oxypurinol treatment after hypoxic-ischemic injury. Pediatr Res 29:362 A(abstr)

41. Spector R 1988 Hypoxanthine transport and metabolism in the central nervous system. J Neurochem 50:969-978

42. Grootveld M. Halliwell B, Moorhouse CP 1987 Action of uric acid, allopurinol and oxypurinol on the myeloperoxidase-derived oxidant hypochlorous acid. Free Rad Res Comms 4:69-76

43. Haberland A. Luther H. Schimke I 1991 Does allopurinol prevent superoxide radical production by xanthine oxidase (XOD)? Agents Actions 32:96-97 